\title{
DIMENSION FREE BOUNDS FOR THE VECTOR-VALUED HARDY-LITTLEWOOD MAXIMAL OPERATOR
}

\author{
LUC DELEAVAL AND CHRISTOPH KRIEGLER
}

\begin{abstract}
In this article, we prove Fefferman-Stein inequalities in $L^{p}\left(\mathbb{R}^{d} ; \ell^{q}\right)$ with bounds independent of the dimension $d$, for all $1<p, q<+\infty$. This result generalizes in a vectorvalued setting the famous one by Stein for the standard Hardy-Littlewood maximal operator. We then extend our result by replacing $\ell^{q}$ with an arbitrary UMD Banach lattice. Finally, we prove similar dimensionless inequalities in the setting of the Grushin operators.
\end{abstract}

\section{INTRODUCTION AND STATEMENT OF THE RESUlTS}

At the beginning of the 1980s, Elias Stein proved in [27] (the complete detailed proof is in the paper of Stein-Strömberg [28]) that the standard Hardy-Littlewood maximal operator, that is associated with Euclidean balls, satisfies $L^{p}\left(\mathbb{R}^{d}\right)$ estimates with constant independent of the dimension $d$ for every $p>1$. More precisely, if we denote by $\mathcal{M}$ the Hardy-Littlewood maximal operator, initially defined for $f \in L_{\text {loc }}^{1}\left(\mathbb{R}^{d}\right)$ by

$$
\mathcal{M} f(x)=\sup _{r>0} \frac{1}{|B(x, r)|} \int_{B(x, r)}|f(y)| d y, \quad x \in \mathbb{R}^{d},
$$

with $B(x, r)$ the Euclidean ball centered at $x$ of radius $r>0$ and $|X|$ the Lebesgue measure of a Borel subset $X$ of $\mathbb{R}^{d}$, then Stein's result reads as follows.

Dimension free bounds for the Hardy-Littlewood maximal operator. Let $1<p \leqslant+\infty$. If $f \in L^{p}\left(\mathbb{R}^{d}\right)$, then we have

$$
\|\mathcal{M} f\|_{L^{p}\left(\mathbb{R}^{d}\right)} \leqslant C(p)\|f\|_{L^{p}\left(\mathbb{R}^{d}\right)},
$$

where $C(p)$ is a constant independent of $d$.

This result, which improves in a spectacular fashion the behavior previously known, has opened the way to the following program: is it possible to bound uniformly in dimension the constant appearing in Hardy-Littlewood type estimates for maximal operators associated with symmetric convex bodies? This topic has been studied by various authors during the period 1986-1990 (see the papers of Bourgain [3, 5, 6, Carbery [8 and Müller 22]), and has been recently renewed by further advances, especially due to Bourgain [7]. For a thorough exposition of this subject, we refer the reader to the recent survey [9. In fact, Stein's result has opened the way, beyond the case of maximal functions, of proving fundamental estimates in harmonic analysis in $\mathbb{R}^{d}$ with formulations with bounds independent of the dimension.

Date: September, 2016.

2010 Mathematics Subject Classification. 42B25; 43A85; $46 \mathrm{~B} 42$.

Key words and phrases. Hardy-Littlewood maximal operator; dimension free bounds; vector-valued estimates; UMD Banach lattice; Grushin operator. 
It is therefore quite surprising that the question of a dimensionless behavior of the constant in the vector-valued extensions of the Hardy-Littlewood maximal theorem, the so-called FeffermanStein inequalities [12, has not been tackled. This is the main purpose of our paper. Let us first recall these inequalities.

Fefferman-Stein inequalities. Let $1<p, q<+\infty$ and let $\left(f_{n}\right)_{n \geqslant 1}$ be a sequence of measurable functions defined on $\mathbb{R}^{d}$. If $\left(\sum_{n=1}^{+\infty}\left|f_{n}(\cdot)\right|^{q}\right)^{\frac{1}{q}} \in L^{p}\left(\mathbb{R}^{d}\right)$, then we have

$$
\left\|\left(\sum_{n=1}^{+\infty}\left|\mathcal{M} f_{n}(\cdot)\right|^{q}\right)^{\frac{1}{q}}\right\|_{L^{p}\left(\mathbb{R}^{d}\right)} \leqslant C(d, p, q)\left\|\left(\sum_{n=1}^{+\infty}\left|f_{n}(\cdot)\right|^{q}\right)^{\frac{1}{q}}\right\|_{L^{p}\left(\mathbb{R}^{d}\right)},
$$

where $C(d, p, q)$ is a constant independent of $\left(f_{n}\right)_{n \geqslant 1}$.

The proof given by Fefferman and Stein for their inequalities, mainly based on the CalderónZygmund decomposition (for a weak-type result), the Marcinkiewicz interpolation theorem and a suitable weighted inequality, leads to a constant which growths exponentially with $d$. Another approach, based on Banach-space valued singular integrals 13. (see also 14]), does not achieve this dimensionless goal either. In this paper, we succeed in proving the following dimensionless result.

Theorem 1. Let $1<p, q<+\infty$ and let $\left(f_{n}\right)_{n \geqslant 1}$ be a sequence of measurable functions defined on $\mathbb{R}^{d}$. If $\left(\sum_{n=1}^{+\infty}\left|f_{n}(\cdot)\right|^{q}\right)^{\frac{1}{q}} \in L^{p}\left(\mathbb{R}^{d}\right)$, then we have

$$
\left\|\left(\sum_{n=1}^{+\infty}\left|\mathcal{M} f_{n}(\cdot)\right|^{q}\right)^{\frac{1}{q}}\right\|_{L^{p}\left(\mathbb{R}^{d}\right)} \leqslant C(p, q)\left\|\left(\sum_{n=1}^{+\infty}\left|f_{n}(\cdot)\right|^{q}\right)^{\frac{1}{q}}\right\|_{L^{p}\left(\mathbb{R}^{d}\right)},
$$

where $C(p, q)$ is a constant independent of $d$ and $\left(f_{n}\right)_{n \geqslant 1}$.

We believe that dimension free vector-valued estimates for general symmetric convex bodies $B$ should be true as well, but certainly not in full generality for both $p$ and $B$. Sharp vector-valued estimates on maximal operators associated with (radial) Fourier multipliers might be a key step, among others, to obtain such dimension free bounds.

Let $\mathcal{S}\left(\mathbb{R}^{d}\right)$ be the Schwartz class of smooth functions $\phi$ such that $\left(1+|x|^{k}\right) \phi^{(l)}(x)$ is bounded on $\mathbb{R}^{d}$ for all integers $k, l \geqslant 0$. As in the proof of the dimensionless result by Stein for the Hardy-Littlewood maximal operator, the main tool in our proof will be the following spherical maximal operator $\mathcal{M}_{S}$, initially defined for $f \in \mathcal{S}\left(\mathbb{R}^{d}\right)$ by

$$
\mathcal{M}_{S} f(x)=\sup _{r>0}\left|\int_{S^{d-1}} f(x-r y) d \sigma(y)\right|, \quad x \in \mathbb{R}^{d},
$$

where $d \sigma$ denotes the normalized Haar measure on $S^{d-1}$, and for which we will prove in particular the following vector-valued estimates.

Theorem 2. Let $d \geqslant 3$ and let $\frac{d}{d-1}<p, q<d$. Let $\left(f_{n}\right)_{n \geqslant 1}$ be a sequence of measurable functions defined on $\mathbb{R}^{d}$. If $\left(\sum_{n=1}^{+\infty}\left|f_{n}(\cdot)\right|^{q}\right)^{\frac{1}{q}} \in L^{p}\left(\mathbb{R}^{d}\right)$, then we have

$$
\left\|\left(\sum_{n=1}^{+\infty}\left|\mathcal{M}_{S} f_{n}(\cdot)\right|^{q}\right)^{\frac{1}{q}}\right\|_{L^{p}\left(\mathbb{R}^{d}\right)} \leqslant C(d, p, q)\left\|\left(\sum_{n=1}^{+\infty}\left|f_{n}(\cdot)\right|^{q}\right)^{\frac{1}{q}}\right\|_{L^{p}\left(\mathbb{R}^{d}\right)},
$$

where $C(d, p, q)$ is a constant independent of $\left(f_{n}\right)_{n \geqslant 1}$.

We point out that vector-valued estimates for $\mathcal{M}_{S}$ have been recently proved by Manna in [20], for the range $\frac{2 d}{d-1}<p, q<+\infty$, by use of a convenient weighted inequality for $\mathcal{M}_{S}$. We 
believe that the range $\frac{d}{d-1}<p, q<+\infty$ is optimal for $d \geqslant 3$, and, also in the case $d=2$, this might be true, as in the scalar case, see [4].

In fact, we shall prove a more general result than Theorem 1. In order to state it, let us recall that a Banach space $Y$ is called UMD space if the Hilbert transform

$$
H: L^{p}(\mathbb{R}) \rightarrow L^{p}(\mathbb{R}), H f(x)=P V-\int_{\mathbb{R}} \frac{1}{x-y} f(y) d y,
$$

extends to a bounded operator on $L^{p}(\mathbb{R} ; Y)$, for some $1<p<+\infty$ (see Section 5 for more details). Here and in what follows, we denote $L^{p}\left(\mathbb{R}^{d} ; Y\right)$ the Bochner-Lebesgue space, i.e. the space of (equivalence classes of) measurable functions $f: \mathbb{R}^{d} \rightarrow Y$ such that

$$
\|f\|_{L^{p}\left(\mathbb{R}^{d} ; Y\right)}^{p}=\int_{\mathbb{R}^{d}}\|f(x)\|_{Y}^{p} d x<+\infty .
$$

With the above reminder in mind, we can now state our second main result, which is concerned with the UMD lattice valued Hardy-Littlewood maximal operator $\mathcal{M}$ (see Section 5 for the precise definition).

Theorem 3. Let $1<p<+\infty$ and $Y=Y(\Omega, \mu)$ be a UMD Banach lattice, consisting without loss of generality of measurable functions over $(\Omega, \mu)$. We have with notations $x \in \mathbb{R}^{d}$ and $y \in \Omega$,

$$
\|\| \mathcal{M}(f(\cdot, y))(x)\left\|_{Y}\right\|_{L^{p}\left(\mathbb{R}^{d}\right)} \leq C(p, Y)\|f\|_{L^{p}\left(\mathbb{R}^{d} ; Y\right)},
$$

where $C(p, Y)$ is a constant independent of $d$ and $f \in L^{p}\left(\mathbb{R}^{d} ; Y\right)$.

The above theorem contains as a particular case Theorem 1 since $\ell^{q}$ is a UMD Banach lattice for $1<q<+\infty$, but we have made the decision, for the reader's convenience, to first prove Theorem 1 which is certainly an enlightening step for readers not familiar with UMD Banach lattices.

As a consequence of our two previous theorems, we shall prove, in the setting of Grushin operators, vector-valued dimension free estimates for both the maximal operator $\mathcal{M}_{C C}$ associated with the Carnot-Carathéodory distance and the maximal operator $\mathcal{M}_{K}$ associated with the Korányi pseudo-distance (see the final section for more details).

Theorem 4. Let $1<p, q<+\infty$. Then $\mathcal{M}_{C C}$ and $\mathcal{M}_{K}$ extend to bounded operators on $L^{p}\left(\mathbb{R}^{d+1} ; \ell^{q}\right)$ and there exists a constant $C=C(p, q)$ independent of $d$ such that

$$
\left\|\left(\sum_{n=1}^{+\infty}\left|\mathcal{M}_{C C} f_{n}(\cdot)\right|^{q}\right)^{\frac{1}{q}}\right\|_{L^{p}\left(\mathbb{R}^{d+1}\right)} \leqslant C(p, q)\left\|\left(\sum_{n=1}^{+\infty}\left|f_{n}(\cdot)\right|^{q}\right)^{\frac{1}{q}}\right\|_{L^{p}\left(\mathbb{R}^{d+1}\right)}
$$

and

$$
\left\|\left(\sum_{n=1}^{+\infty}\left|\mathcal{M}_{K} f_{n}(\cdot)\right|^{q}\right)^{\frac{1}{q}}\right\|_{L^{p}\left(\mathbb{R}^{d+1}\right)} \leqslant C(p, q)\left\|\left(\sum_{n=1}^{+\infty}\left|f_{n}(\cdot)\right|^{q}\right)^{\frac{1}{q}}\right\|_{L^{p}\left(\mathbb{R}^{d+1}\right)} .
$$

In the same manner, if $Y$ is a UMD Banach lattice, then $\mathcal{M}_{C C}$ and $\mathcal{M}_{K}$ extend to bounded operators on $L^{p}\left(\mathbb{R}^{d+1} ; Y\right)$ with norm $C=C(p, Y)$ independent of $d$.

We end this introduction with an overview of the sections. In Section 2 we prove two preliminary results on vector-valued maximal operators associated with multipliers and a Hilbertian square function estimate that we need in the sequel. The method of proof of the main Theorems 11 and 3 uses vector-valued estimates for the spherical maximal operator. The latter is then studied in Section 3, and both a sharp Hilbertian $L^{2}\left(\mathbb{R}^{d} ; \ell^{2}\right)$ estimate and a weaker $L^{p}\left(\mathbb{R}^{d} ; \ell^{q}\right)$ estimate are built together by means of complex interpolation to yield the desired spherical 
maximal operator estimates, stated in Theorem [2. The next two Sections 4 and 5 are devoted to the proofs of Theorems 1 and 3 respectively. They use a technique of descent in the spirit of the Calderón-Zygmund method of rotations, and the spherical maximal operator estimates established beforehand. Low dimensional estimates in the general case of UMD-lattice valued $L^{p}$ spaces are covered by the recent work of $\mathrm{Xu} \mathrm{[30.} \mathrm{Finally,} \mathrm{in} \mathrm{Section} \mathrm{[6]} \mathrm{we} \mathrm{will} \mathrm{prove} \mathrm{Theorem} 4$.

\section{Preliminary Results}

For $\omega \in \mathcal{S}\left(\mathbb{R}^{d}\right)$ a radial function, we shall denote by $M_{\omega}$ the maximal operator associated with the multiplier $\omega$ and initially defined for $f \in \mathcal{S}\left(\mathbb{R}^{d}\right)$ by

$$
M_{\omega} f(x)=\sup _{r>0}\left|(\hat{f}(\cdot) \omega(r \cdot))^{\vee}(x)\right|=\sup _{r>0}\left|\left(f *\left(\omega^{\vee}\right)_{r}\right)(x)\right|, \quad x \in \mathbb{R}^{d},
$$

where ${ }^{\vee}$ is the inverse Fourier transform and where, for suitable $\psi, \psi_{r}$ is the dilation of $\psi$, that is to say

$$
\psi_{r}(x)=\frac{1}{r^{d}} \psi\left(\frac{x}{r}\right), \quad x \in \mathbb{R}^{d} .
$$

The following proposition provides us Fefferman-Stein inequalities for maximal operators associated with such a multiplier $\omega$.

Proposition 1. Let $1<p, q<+\infty$ and let $\left(f_{n}\right)_{n \geqslant 1}$ be a sequence of measurable functions defined on $\mathbb{R}^{d}$. If $\left(\sum_{n=1}^{+\infty}\left|f_{n}(\cdot)\right|^{q}\right)^{\frac{1}{q}} \in L^{p}\left(\mathbb{R}^{d}\right)$, then we have

$$
\left\|\left(\sum_{n=1}^{+\infty}\left|M_{\omega} f_{n}(\cdot)\right|^{q}\right)^{\frac{1}{q}}\right\|_{L^{p}\left(\mathbb{R}^{d}\right)} \leqslant C(d, p, q)\left\|\left(\sum_{n=1}^{+\infty}\left|f_{n}(\cdot)\right|^{q}\right)^{\frac{1}{q}}\right\|_{L^{p}\left(\mathbb{R}^{d}\right)},
$$

where $C(d, p, q)$ is a constant independent of $\left(f_{n}\right)_{n \geqslant 1}$.

Proof. We claim that $\omega$ has an integrable radially decreasing majorant $\Omega$ since $\omega^{\vee}$ is a Schwartz radial function. Therefore, we have (see Corollary 2.1.12. page 84 in [14]) for every $x \in \mathbb{R}^{d}$

$$
M_{\omega} f(x)=\sup _{r>0}\left|\left(f *\left(\omega^{\vee}\right)_{r}\right)(x)\right| \leqslant\|\Omega\|_{L^{1}\left(\mathbb{R}^{d}\right)} \mathcal{M} f(x) .
$$

Thus, all we have to do to conclude is to use the standard version of the vector-valued estimates for the Hardy-Littlewood maximal operator.

We point out that the proof above applies to the following weak-type result: if $1<q<+\infty$ and if $\left(\sum_{n=1}^{+\infty}\left|f_{n}(\cdot)\right|^{q}\right)^{\frac{1}{q}} \in L^{1}\left(\mathbb{R}^{d}\right)$, then for every $\lambda>0$ we have

$$
\left|\left\{x \in \mathbb{R}^{d}:\left(\sum_{n=1}^{+\infty}\left|M_{\omega} f_{n}(x)\right|^{q}\right)^{\frac{1}{q}}>\lambda\right\}\right| \leqslant \frac{C(d, q)}{\lambda}\left\|\left(\sum_{n=1}^{+\infty}\left|f_{n}(\cdot)\right|^{q}\right)^{\frac{1}{q}}\right\|_{L^{1}\left(\mathbb{R}^{d}\right)},
$$

where $C(d, q)$ is a constant independent of $\left(f_{n}\right)_{n \geqslant 1}$ and $\lambda$.

We now introduce a square function that is closely related to the previous maximal multiplier operator. For a (radial) function $\omega \in \mathcal{S}\left(\mathbb{R}^{d}\right)$, we denote by $g_{\omega}$ the square function associated with the multiplier $\omega$ and initially defined for $f \in \mathcal{S}\left(\mathbb{R}^{d}\right)$ by

$$
g_{\omega}(f)(x)=\left(\int_{0}^{+\infty}\left|(\hat{f}(x) \omega(t x))^{\vee}\right|^{2} \frac{d t}{t}\right)^{\frac{1}{2}}, \quad x \in \mathbb{R}^{d} .
$$

If the multiplier $\omega$ is supported in an annulus, then we can give the following precise upper bound, where a Hilbertian structure is required. 
Proposition 2. Let $r$ be a positive real number. Suppose that $\omega$ is supported in the annulus $\left\{x \in \mathbb{R}^{d}: r \leqslant|x| \leqslant \rho r\right\}$ (with $\rho>1$ ) and is bounded by $C$. Let $\left(f_{n}\right)_{n \geqslant 1}$ be a sequence of measurable functions defined on $\mathbb{R}^{d}$. If $\left(\sum_{n=1}^{+\infty}\left|f_{n}(\cdot)\right|^{2}\right)^{\frac{1}{2}} \in L^{2}\left(\mathbb{R}^{d}\right)$, then we have

$$
\left\|\left(\sum_{n=1}^{+\infty}\left|g_{\omega}\left(f_{n}\right)(\cdot)\right|^{2}\right)^{\frac{1}{2}}\right\|_{L^{2}\left(\mathbb{R}^{d}\right)} \leqslant C \sqrt{\ln (\rho)}\left\|\left(\sum_{n=1}^{+\infty}\left|f_{n}(\cdot)\right|^{2}\right)^{\frac{1}{2}}\right\|_{L^{2}\left(\mathbb{R}^{d}\right)},
$$

where $C$ is the same constant in both the hypothesis and conclusion of the proposition.

Proof. The proof is nearly obvious. Indeed, by using successively Fubini's theorem, Plancherel's theorem and Fubini's theorem again, we have

$$
\int_{\mathbb{R}^{d}} \sum_{n=1}^{+\infty}\left|g_{\omega}\left(f_{n}\right)(x)\right|^{2} d x=\sum_{n=1}^{+\infty} \int_{\mathbb{R}^{d}}\left(\int_{0}^{+\infty}\left|\hat{f}_{n}(x) \omega(t x)\right|^{2} \frac{d t}{t}\right) d x
$$

For all $x \in \mathbb{R}^{d} \backslash\{0\}$ and all $n \geqslant 1$, we can write

$$
\int_{0}^{+\infty}\left|\hat{f}_{n}(x) \omega(t x)\right|^{2} \frac{d t}{t}=\left|\hat{f}_{n}(x)\right|^{2} \int_{\frac{r}{|x|}}^{\frac{\rho r}{x \mid}}|\omega(t x)|^{2} \frac{d t}{t} \leqslant C^{2} \ln (\rho)\left|\hat{f}_{n}(x)\right|^{2},
$$

thus

$$
\int_{\mathbb{R}^{d}} \sum_{n=1}^{+\infty}\left|g_{\omega}\left(f_{n}\right)(x)\right|^{2} d x \leqslant C^{2} \ln (\rho) \sum_{n=1}^{+\infty} \int_{\mathbb{R}^{d}}\left|\hat{f}_{n}(x)\right|^{2} d x .
$$

To conclude, it is now enough to use Plancherel's theorem and Fubini's theorem.

\section{VECTOR-VALUED INEQUALITES FOR THE SPHERICAL MAXIMAL OPERATOR}

In this section, we prove the vector-valued inequalities for the spherical maximal operator, stated in Theorem 2. These estimates will be a key tool in the proof of our dimensionless results. Let us begin with the following remark.

Remark. The condition $\frac{d}{d-1}<p$ can be easily seen to be necessary. Indeed, it suffices to consider the following sequence

$$
f_{1}(x)=\left\{\begin{array}{l}
\mathrm{e}^{\frac{-1}{1-|x|^{2}}} \text { if }|x|<1 \quad f_{2}=f_{3}=\ldots=0 . \\
0 \text { if }|x| \geqslant 1
\end{array}\right.
$$

Of course, $\left(\sum_{n=1}^{+\infty}\left|f_{n}(\cdot)\right|^{q}\right)^{\frac{1}{q}} \in L^{p}\left(\mathbb{R}^{d}\right)$ while

$$
\left(\sum_{n=1}^{+\infty}\left|\mathcal{M}_{S} f_{n}(\cdot)\right|^{q}\right)^{\frac{1}{q}} \notin L^{p}\left(\mathbb{R}^{d}\right)
$$

for $p \leqslant \frac{d}{d-1}$ since for $|x|$ large enough,

$$
\left(\sum_{n=1}^{+\infty}\left|\mathcal{M}_{S} f_{n}(x)\right|^{q}\right)^{\frac{1}{q}} \geqslant \frac{C(d)}{|x|^{d-1}} .
$$

However, the condition $p<d$ is not optimal. Indeed, in a recent paper, Manna has proved by means of a convenient weighted inequality that Theorem 2 is true for the range $\frac{2 d}{d-1}<p, q<+\infty$.

In order to prove Theorem 2, we do not follow Stein's ideas for the scalar case, but rather those of Rubio de Francia in [23]. More precisely, we shall dominate $\mathcal{M}_{S}$ by a series of maximal multiplier operators $\mathcal{M}_{S} \leqslant \sum_{l=0}^{+\infty} M_{m_{l}}$ (where $m_{l}$ is a radial multiplier), and we shall establish, for each $M_{m_{l}}$, a sharp $L^{2}\left(\mathbb{R}^{d} ; \ell^{2}\right)$ estimate and a weaker $L^{p}\left(\mathbb{R}^{d} ; \ell^{q}\right)$ estimate. Then, we shall 
proceed by complex interpolation, and the range of $p, q$, in Theorem 2 is then relevant for series convergence. For the $L^{2}\left(\mathbb{R}^{d} ; \ell^{2}\right)$ case, we mainly use both the decay at infinity and a support property for $m_{l}$, and a precise upper bound for the $L^{2}\left(\mathbb{R}^{d}\right)$-norm of an associated square function. For the $L^{p}\left(\mathbb{R}^{d} ; \ell^{q}\right)$ case, we mainly use the standard Fefferman-Stein inequalities and the FunkHecke formula. For the reader's convenience, we shall give the complete detailed proofs, which owe a lot to $9,14,23$.

To begin, we note that the spherical maximal operator could be expressed as follows

$$
\mathcal{M}_{S} f(x)=\sup _{r>0}\left|(\hat{f}(\cdot) m(r \cdot))^{\vee}(x)\right|=\sup _{r>0}\left|\left(f *\left(m^{\vee}\right)_{r}\right)(x)\right|,
$$

where the multiplier $m$ is given by

$$
m(x)=\widehat{d \sigma}(x)=\frac{2 \pi}{|x|^{\frac{d-2}{2}}} J_{\frac{d-2}{2}}(2 \pi|x|),
$$

with $J_{\alpha}$ the Bessel function of order $\alpha$. In order to decompose this multiplier into radial pieces with localized frequencies, we consider a smooth radial function $\varphi_{0}$ on $\mathbb{R}^{d}$ satisfying

$$
\varphi_{0}(x)=\left\{\begin{array}{l}
1 \text { if }|x| \leqslant 1 \\
0 \text { if }|x| \geqslant 2
\end{array}\right.
$$

Then, for every positive integer $l$, we define

$$
\varphi_{l}(x)=\varphi_{0}\left(2^{-l} x\right)-\varphi_{0}\left(2^{1-l} x\right),
$$

and we therefore introduce the following dyadic radial pieces associated with the multiplier $m$

$$
\forall l \geqslant 0, \quad m_{l}=\varphi_{l} m .
$$

Since it is obvious that $\sum_{l=0}^{+\infty} \varphi_{l}=1$, we claim that

$$
m=\sum_{l=0}^{+\infty} m_{l} .
$$

Consequently, we have the following pointwise inequality

$$
\mathcal{M}_{S} f(x) \leqslant \sum_{l=0}^{+\infty} M_{m_{l}} f(x), \quad x \in \mathbb{R}^{d}
$$

where $M_{m_{l}}$ is defined at the beginning of Section 2 by specializing $\omega$ to $m_{l}$.

$L^{2}\left(\mathbb{R}^{d} ; \ell^{2}\right)$ and $L^{p}\left(\mathbb{R}^{d} ; \ell^{q}\right)$ estimates for $M_{m_{l}}$. As claimed before, we shall establish an $L^{2}\left(\mathbb{R}^{d} ; \ell^{2}\right)$ estimate and an $L^{p}\left(\mathbb{R}^{d} ; \ell^{q}\right)$ estimate for $M_{m_{l}}$, wtih $l \geqslant 1$. As we shall see in the proof of Theorem 2. the case $M_{m_{0}}$ will be covered by Proposition 1.

Let us begin with the $L^{2}\left(\mathbb{R}^{d} ; \ell^{2}\right)$-result for $M_{m_{l}}$.

Proposition 3. Let $l \geqslant 1$ and let $\left(f_{n}\right)_{n \geqslant 1}$ be a sequence of measurable functions defined on $\mathbb{R}^{d}$. If $\left(\sum_{n=1}^{+\infty}\left|f_{n}(\cdot)\right|^{2}\right)^{\frac{1}{2}} \in L^{2}\left(\mathbb{R}^{d}\right)$, then we have

$$
\left\|\left(\sum_{n=1}^{+\infty}\left|M_{m_{l}} f_{n}(\cdot)\right|^{2}\right)^{\frac{1}{2}}\right\|_{L^{2}\left(\mathbb{R}^{d}\right)} \leqslant \frac{C(d)}{2^{\frac{l(d-2)}{2}}}\left\|\left(\sum_{n=1}^{+\infty}\left|f_{n}(\cdot)\right|^{2}\right)^{\frac{1}{2}}\right\|_{L^{2}\left(\mathbb{R}^{d}\right)},
$$

where $C(d)$ is a constant independent of $l$ and $\left(f_{n}\right)_{n \geqslant 1}$. 
Proof. Let $n \geqslant 1$ and $l \geqslant 1$. By applying the well-known differentiation theorem for multiples of approximate identities (see Corollary 2.1.19. page 88 in [14]), we get for almost all $x \in \mathbb{R}^{d}$

$$
\left(f_{n} *\left(m_{l}^{\vee}\right)_{r}\right)(x) \rightarrow m_{l}(0) f_{n}(x)=0
$$

as $r$ goes to 0 . We can therefore write for almost $x \in \mathbb{R}^{d}$

$$
\begin{aligned}
\left(f_{n} *\left(m_{l}^{\vee}\right)_{r}\right)^{2}(x) & =\int_{0}^{r} \frac{d}{d t}\left(\left(f_{n} *\left(m_{l}^{\vee}\right)_{t}\right)^{2}(x)\right) d t \\
& =2 \int_{0}^{r}\left(f_{n} *\left(m_{l}^{\vee}\right)_{t}\right)(x)\left(f_{n} *\left(\tilde{m}_{l}^{\vee}\right)_{t}\right)(x) \frac{d t}{t},
\end{aligned}
$$

where we have set

$$
\tilde{m}_{l}(x)=\left\langle x, \nabla m_{l}(x)\right\rangle
$$

We now enlarge the domain of the integral to obtain

$$
\left|\left(f_{n} *\left(m_{l}^{\vee}\right)_{r}\right)(x)\right|^{2} \leqslant 2 \int_{0}^{+\infty}\left|\left(f_{n} *\left(m_{l}^{\vee}\right)_{t}\right)(x)\right|\left|\left(f_{n} *\left(\tilde{m}_{l}^{\vee}\right)_{t}\right)(x)\right| \frac{d t}{t}
$$

and this previous inequality can be reformulated as follows

$$
\left|\left(\hat{f}_{n}(\cdot) m_{l}(r \cdot)\right)^{\vee}(x)\right|^{2} \leqslant 2 \int_{0}^{+\infty}\left|\left(\hat{f}_{n}(\cdot) m_{l}(t \cdot)\right)^{\vee}(x)\right|\left|\left(\hat{f}_{n}(\cdot) \tilde{m}_{l}(t \cdot)\right)^{\vee}(x)\right| \frac{d t}{t} .
$$

We first take the supremum over all $r>0$ and then use the Cauchy-Schwarz inequality in order to get

$$
\left(M_{m_{l}} f_{n}(x)\right)^{2} \leqslant 2 g_{m_{l}}\left(f_{n}\right)(x) g_{\tilde{m}_{l}}\left(f_{n}\right)(x) .
$$

By summing over $n$ and by using again the Cauchy-Schwarz inequality, we are led to

$$
\sum_{n=1}^{+\infty}\left|M_{m_{l}} f_{n}(x)\right|^{2} \leqslant 2\left(\sum_{n=1}^{+\infty}\left|g_{m_{l}}\left(f_{n}\right)(x)\right|^{2}\right)^{\frac{1}{2}}\left(\sum_{n=1}^{+\infty}\left|g_{\tilde{m}_{l}}\left(f_{n}\right)(x)\right|^{2}\right)^{\frac{1}{2}} .
$$

We now integrate over $\mathbb{R}^{d}$ and we use the Cauchy-Schwarz inequality to deduce that

$$
\begin{aligned}
\left\|\left(\sum_{n=1}^{+\infty}\left|M_{m_{l}} f_{n}(\cdot)\right|^{2}\right)^{\frac{1}{2}}\right\|_{L^{2}\left(\mathbb{R}^{d}\right)}^{2} \leqslant & \\
& 2\left\|\left(\sum_{n=1}^{+\infty}\left|g_{m_{l}}\left(f_{n}\right)(\cdot)\right|^{2}\right)^{\frac{1}{2}}\right\|_{L^{2}\left(\mathbb{R}^{d}\right)}\left\|\left(\sum_{n=1}^{+\infty}\left|g_{\tilde{m}_{l}}\left(f_{n}\right)(\cdot)\right|^{2}\right)^{\frac{1}{2}}\right\|_{L^{2}\left(\mathbb{R}^{d}\right)} .
\end{aligned}
$$

Let us note the following immediate inclusions

$$
\begin{aligned}
& \operatorname{supp}\left(m_{l}\right) \subset\left\{x \in \mathbb{R}^{d}: 2^{l-1} \leqslant|x| \leqslant 2^{l+1}\right\} \\
& \operatorname{supp}\left(\tilde{m}_{l}\right) \subset\left\{x \in \mathbb{R}^{d}: 2^{l-1} \leqslant|x| \leqslant 2^{l+1}\right\} .
\end{aligned}
$$

Therefore, thanks to Proposition 2, it is now enough to prove the following inequalities

$$
\left\|m_{l}\right\|_{L^{\infty}\left(\mathbb{R}^{d}\right)} \leqslant \frac{C_{1}(d)}{2^{\frac{l(d-1)}{2}}}, \quad\left\|\tilde{m}_{l}\right\|_{L^{\infty}\left(\mathbb{R}^{d}\right)} \leqslant \frac{C_{2}(d)}{2^{\frac{l(d-3)}{2}}},
$$


where both $C_{1}(d)$ and $C_{2}(d)$ are constants independent of $l$, since we shall deduce

$$
\begin{aligned}
&\left\|\left(\sum_{n=1}^{+\infty}\left|M_{m_{l}} f_{n}(\cdot)\right|^{2}\right)^{\frac{1}{2}}\right\|_{L^{2}\left(\mathbb{R}^{d}\right)}^{2} \leqslant \\
& 2\left(\frac{C_{1}(d) \sqrt{\ln 4}}{2^{\frac{l(d-1)}{2}}}\left\|\left(\sum_{n=1}^{+\infty}\left|f_{n}(\cdot)\right|^{2}\right)^{\frac{1}{2}}\right\|_{L^{2}\left(\mathbb{R}^{d}\right)}\right)\left(\frac{C_{2}(d) \sqrt{\ln 4}}{2^{\frac{l(d-3)}{2}}}\left\|\left(\sum_{n=1}^{+\infty}\left|f_{n}(\cdot)\right|^{2}\right)^{\frac{1}{2}}\right\|_{L^{2}\left(\mathbb{R}^{d}\right)}\right) .
\end{aligned}
$$

Thus, we now turn to the proof of (2) in order to complete the proof of the proposition. The following well-known estimate for the Bessel function (see for instance page 238 in [2])

$$
\sup _{x \geqslant 0} x^{1 / 2}\left|J_{\alpha}(x)\right|<+\infty
$$

together with the following equality

$$
\frac{d}{d t} J_{\alpha}(t)=\frac{1}{2}\left(J_{\alpha-1}(t)-J_{\alpha+1}(t)\right)
$$

allow us to write for all $x \in \mathbb{R}^{d} \backslash\{0\}$

$$
\left|m_{l}(x)\right| \leqslant \frac{C_{1}(d)}{|x|^{\frac{d-1}{2}}}, \quad\left|\tilde{m}_{l}(x)\right| \leqslant \frac{C_{2}(d)}{|x|^{\frac{d-3}{2}}} .
$$

We claim that (2) is proved since both $m_{l}$ and $\tilde{m}_{l}$ are localized near $|x| \simeq 2^{l}$.

We now turn to a weaker $L^{p}\left(\mathbb{R}^{d} ; \ell^{q}\right)$-result for $M_{m_{l}}$.

Proposition 4. Let $l \geqslant 1$ and let $1<p, q<+\infty$. Let $\left(f_{n}\right)_{n \geqslant 1}$ be a sequence of measurable functions defined on $\mathbb{R}^{d}$. If $\left(\sum_{n=1}^{+\infty}\left|f_{n}(\cdot)\right|^{q}\right)^{\frac{1}{q}} \in L^{p}\left(\mathbb{R}^{d}\right)$, then we have

$$
\left\|\left(\sum_{n=1}^{+\infty}\left|M_{m_{l}} f_{n}(\cdot)\right|^{q}\right)^{\frac{1}{q}}\right\|_{L^{p}\left(\mathbb{R}^{d}\right)} \leqslant C(d, p, q) 2^{l}\left\|\left(\sum_{n=1}^{+\infty}\left|f_{n}(\cdot)\right|^{q}\right)^{\frac{1}{q}}\right\|_{L^{p}\left(\mathbb{R}^{d}\right)},
$$

where $C(d, p, q)$ is a constant independent of $l$ and $\left(f_{n}\right)_{n \geqslant 1}$.

Proof. If we prove that for all $x \in \mathbb{R}^{d}$ and $l \geqslant 1$,

$$
\left|m_{l}^{\vee}(x)\right| \leqslant C(d) \frac{2^{l}}{(1+|x|)^{d+1}}
$$

then we claim, thanks to Corollary 2.1.12. page 84 in [14], that

$$
\sup _{r>0}\left|\left(f_{n} *\left(m_{l}^{\vee}\right)_{r}\right)(x)\right| \leqslant \tilde{C}(d) 2^{l} \mathcal{M} f_{n}(x),
$$

and the standard Fefferman-Stein inequalities for the Hardy-Littlewood maximal operator $\mathcal{M}$ allows us to conclude. Therefore, we are left with the task of establishing (3) to which we now turn. Let $x \in \mathbb{R}^{d}$. We can write by use of the Funk-Hecke formula

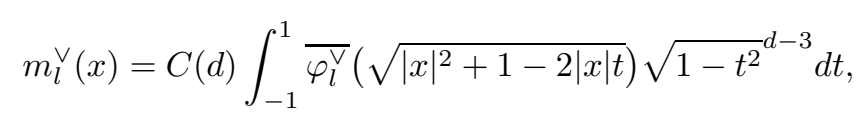

where we have used the notation, for a radial function $f$ and for all $\xi \in \mathbb{R}^{d}, f(\xi)=\bar{f}(|\xi|)$. Since we have $\varphi_{l}^{\vee}=\Psi_{2^{-l}}$, with $\Psi \in \mathcal{S}\left(\mathbb{R}^{d}\right)$ by the very definition of $\varphi_{l}$, then

$$
\left|m_{l}^{\vee}(x)\right| \leqslant C(d) \int_{-1}^{1} \frac{2^{d l}}{\left(1+2^{l}{\sqrt{|x|^{2}+1-2|x| t}}^{d+2}\right.}{\sqrt{1-t^{2}}}^{d-3} d t .
$$


We set

$$
\begin{aligned}
& I_{-1}(x)=[-1,1] \cap\left\{t \in \mathbb{R}: \sqrt{|x|^{2}+1-2|x| t} \leqslant 2^{-l}\right\} \\
& I_{j}(x)=[-1,1] \cap\left\{t \in \mathbb{R}: 2^{j-l}<\sqrt{|x|^{2}+1-2|x| t} \leqslant 2^{j+1-l}\right\}, \quad \forall j \geqslant 0,
\end{aligned}
$$

in order to write

$$
\left|m_{l}^{\vee}(x)\right| \leqslant C(d)\left(\Sigma_{1}+\Sigma_{2}\right)
$$

with

$$
\begin{aligned}
& \Sigma_{1}=\sum_{j=-1}^{l} \int_{I_{j}(x)} \frac{2^{d l}}{\left(1+2^{l} \sqrt{|x|^{2}+1-2|x| t}\right)^{d+2}}{\sqrt{1-t^{2}}}^{d-3} d t \\
& \Sigma_{2}=\sum_{j=l+1}^{+\infty} \int_{I_{j}(x)} \frac{2^{d l}}{\left(1+2^{l} \sqrt{|x|^{2}+1-2|x| t}\right)^{d+2}}{\sqrt{1-t^{2}}}^{d-3} d t .
\end{aligned}
$$

Therefore, the inequality (3) is true if we show that

$$
\Sigma_{1} \leqslant C_{1}(d) \frac{2^{l}}{(1+|x|)^{d+1}}, \quad \Sigma_{2} \leqslant C_{2}(d) \frac{2^{l}}{(1+|x|)^{d+1}},
$$

where both $C_{1}(d)$ and $C_{2}(d)$ are constants independent of $l$. First, let us remark that for $t \in I_{j}(x)$, $j \geqslant-1$, we have

$$
|x| \leqslant 2^{j+1-l}+1
$$

since for $x \in \mathbb{R}^{d}$ and $t \in I_{j}(x)$ fixed, we have

$$
\begin{aligned}
|x| \leqslant \sqrt{(|x|-t)^{2}}+|t| & \leqslant \sqrt{(|x|-t)^{2}+\left(1-t^{2}\right)}+|t| \\
& =\sqrt{|x|^{2}+1-2|x| t}+|t| \leqslant 2^{j+1-l}+1 .
\end{aligned}
$$

We first prove the desired estimate for $\Sigma_{1}$. The following trivial observation $-1 \leqslant j \leqslant l \Longrightarrow$ $2^{j+1-l}+1 \leqslant 3$ together with (4) lead us to

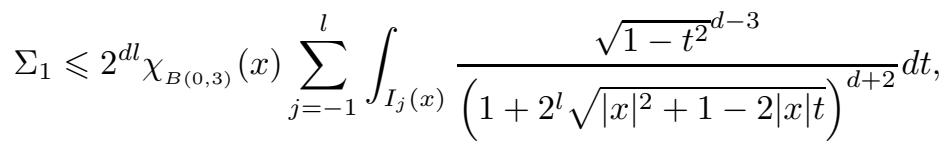

where we denote by $\chi_{X}$ the characteristic function of the set $X$. Moreover, for all $t \in I_{j}(x)$, $0 \leqslant j \leqslant l$

$$
\frac{1}{\left(1+2^{l} \sqrt{|x|^{2}+1-2|x| t}\right)^{d+2}} \leqslant \frac{1}{2^{j(d+2)}}
$$

and, since this inequality remains obviously true for $j=-1$, we obtain

$$
\Sigma_{1} \leqslant 2^{d l} \chi_{B(0,3)}(x) \sum_{j=-1}^{l}\left(\frac{1}{2^{j(d+2)}} \int_{I_{j}(x)}{\sqrt{1-t^{2}}}^{d-3} d t\right) .
$$

Now we claim that, for all $-1 \leqslant j \leqslant l$,

$$
\int_{I_{j}(x)}{\sqrt{1-t^{2}}}^{d-3} d t \leqslant 2^{(j+1-l)(d-1)+1} .
$$


Indeed, we have

$$
\begin{aligned}
& \int_{I_{j}(x)}{\sqrt{1-t^{2}}}^{d-3} d t \leqslant \int_{[-1,1] \cap\left\{t \in \mathbb{R}: \sqrt{1-t^{2}} \leqslant 2^{j+1-l}\right\}}{\sqrt{1-t^{2}}}^{d-3} d t \\
& \leqslant 2^{(j+1-l)(d-3)} \int_{[-1,1] \cap\left\{t \in \mathbb{R}: \sqrt{1-|t|} \leqslant 2^{j+1-l}\right\}} d t,
\end{aligned}
$$

and the following obvious observation

$$
t \in[-1,1] \cap\left\{t \in \mathbb{R}: \sqrt{1-|t|} \leqslant 2^{j+1-l}\right\} \Longrightarrow 1-2^{2(j+1-l)} \leqslant|t| \leqslant 1
$$

then leads us to

$$
\int_{I_{j}(x)}{\sqrt{1-t^{2}}}^{d-3} d t \leqslant 2^{(j+1-l)(d-3)+1} \int_{1-2^{2(j+1-l)}}^{1} d t=2^{(j+1-l)(d-1)+1} .
$$

Consequently, we have

$$
\Sigma_{1} \leqslant 2 \sum_{j=-1}^{l} \frac{2^{d l} 2^{(j+1-l)(d-1)}}{2^{j(d+2)}} \chi_{B(0,3)}(x)=2^{d+l} \sum_{j=-1}^{l} \frac{\chi_{B(0,3)}(x)}{2^{3 j}} \leqslant C(d) \frac{2^{l}}{(1+|x|)^{d+1}},
$$

and it remains to prove the same estimate for $\Sigma_{2}$.

Thanks to (4) and to the very definition of $I_{j}(x)$, we claim that

$$
\Sigma_{2} \leqslant 2^{d l} \sum_{j=l+1}^{+\infty} \frac{\chi_{B\left(0,2^{j+1-l}+1\right)}(x)}{2^{j(d+2)}} \int_{I_{j}(x)}{\sqrt{1-t^{2}}}^{d-3} d t .
$$

The obvious inclusion $B\left(0,2^{j+1-l}+1\right) \subset B\left(0,2^{j+2-l}\right)$, for all $j \geqslant l+1$, together with the fact that

$$
\int_{I_{j}(x)}{\sqrt{1-t^{2}}}^{d-3} d t \leqslant \int_{-1}^{1}{\sqrt{1-t^{2}}}^{d-3} d t \leqslant C(d)
$$

allow us to write

$$
\Sigma_{2} \leqslant C(d) 2^{d l} \sum_{j=l+1}^{+\infty} \frac{\chi_{B\left(0,2^{j+2-l}\right)}(x)}{2^{j(d+2)}} \leqslant \frac{C(d) 2^{d l}}{(1+|x|)^{d+1}} \sum_{j=l+1}^{+\infty} \frac{\left(1+2^{j+2-l}\right)^{d+1}}{2^{j(d+2)}},
$$

from which we deduce that

$$
\begin{aligned}
\Sigma_{2} & \leqslant \frac{C(d) 2^{l}}{(1+|x|)^{d+1}} \sum_{j=l+1}^{+\infty} \frac{2^{(j-l)((d+1)-(d+2))}}{2^{l((d+2)+1-d)}} \\
& \leqslant \frac{C(d) 2^{l}}{(1+|x|)^{d+1}} \sum_{j=l+1}^{+\infty} \frac{2^{l-j}}{2^{3 l}} \\
& \leqslant C(d) \frac{2^{l}}{(1+|x|)^{d+1}} .
\end{aligned}
$$

Remark. We point out that the pointwise inequality (3), which implies that

$$
\sup _{r>0}\left|\left(f_{n} *\left(m_{l}^{\vee}\right)_{r}\right)(x)\right| \leqslant \tilde{C}(d) 2^{l} \mathcal{M} f_{n}(x),
$$


gives us the following weak-type $L^{1}\left(\mathbb{R}^{d} ; \ell^{q}\right)$-result $(1<q<+\infty)$, with a constant $2^{l}$ : if $\left(\sum_{n=1}^{+\infty}\left|f_{n}(\cdot)\right|^{q}\right)^{\frac{1}{q}} \in L^{1}\left(\mathbb{R}^{d}\right)$, then for every $\lambda>0$ we have

$$
\left|\left\{x \in \mathbb{R}^{d}:\left(\sum_{n=1}^{+\infty}\left|M_{m_{l}} f_{n}(x)\right|^{q}\right)^{\frac{1}{q}}>\lambda\right\}\right| \leqslant C(d, q) \frac{2^{l}}{\lambda}\left\|\left(\sum_{n=1}^{+\infty}\left|f_{n}(\cdot)\right|^{q}\right)^{\frac{1}{q}}\right\|_{L^{1}\left(\mathbb{R}^{d}\right)},
$$

where $C(d, q)$ is a constant independent of $\left(f_{n}\right)_{n \geqslant 1}$ and $\lambda$.

We are now in a position to prove Theorem 2

Proof of Theorem Q We begin the proof by noting that for all $1<p, q<+\infty, l \geqslant 1$ and $\left(f_{n}(\cdot)\right)_{n \geqslant 1} \in L^{p}\left(\mathbb{R}^{d} ; \ell^{q}\right)$, we have

$$
\left\|\left(\sum_{n=1}^{+\infty}\left|M_{m_{l}} f_{n}(\cdot)\right|^{q}\right)^{\frac{1}{q}}\right\|_{L^{p}\left(\mathbb{R}^{d}\right)}=\left\|A_{m_{l}}\left(\left(f_{n}(\cdot)\right)_{n \geqslant 1}\right)\right\|_{L^{p}\left(\mathbb{R}^{d} ; \ell^{q}\left(L^{\infty}(] 0,+\infty[)\right)\right)},
$$

where we have set

$$
A_{m_{l}}:\left\{\begin{array}{l}
L^{p}\left(\mathbb{R}^{d} ; \ell^{q}\right) \rightarrow L^{p}\left(\mathbb{R}^{d} ; \ell^{q}\left(L^{\infty}(] 0,+\infty[)\right)\right) \\
\left(f_{n}(\cdot)\right)_{n \geqslant 1} \mapsto\left(r \mapsto f_{n} *\left(m_{l}^{\vee}\right)_{r}\right)_{n \geqslant 1} .
\end{array}\right.
$$

Proposition 3 yields that

$$
\left\|A_{m_{l}}\right\|_{L^{2}\left(\mathbb{R}^{d} ; \ell^{2}\right) \rightarrow L^{2}\left(\mathbb{R}^{d} ; \ell^{2}\left(L^{\infty}(] 0,+\infty[)\right)\right)} \leqslant C(d) 2^{-\frac{l(d-2)}{2}},
$$

whereas Proposition 4 yields

$$
\left\|A_{m_{l}}\right\|_{L^{p_{0}}\left(\mathbb{R}^{d} ; \ell^{q_{0}}\right) \rightarrow L^{p_{0}}\left(\mathbb{R}^{d} ; \ell^{q_{0}}\left(L^{\infty}(] 0,+\infty[)\right)\right)} \leqslant C\left(d, p_{0}, q_{0}\right) 2^{l}
$$

for any $1<p_{0}, q_{0}<+\infty$. Complex interpolation between these two estimates yields for $\frac{1}{p}=$ $\eta \frac{1}{2}+(1-\eta) \frac{1}{p_{0}}$ and $\frac{1}{q}=\eta \frac{1}{2}+(1-\eta) \frac{1}{q_{0}}$ that

$$
\left\|A_{m_{l}}\right\|_{L^{p}\left(\mathbb{R}^{d} ; \ell q\right) \rightarrow L^{p}\left(\mathbb{R}^{d} ; \ell q\left(L^{\infty}(] 0,+\infty[)\right)\right)} \leqslant C(d, p, q) 2^{l\left(1-\frac{d}{2} \eta\right)} .
$$

Note that for given $\frac{d}{d-1}<p, q<d$, we can choose $\left.\eta \in\right] 0,1[$ such that

$$
\frac{1}{d}<\frac{\eta}{2}<\frac{1}{p}, \frac{1}{q}<1-\frac{\eta}{2}<\frac{d-1}{d} .
$$

The second and third inequality yield that the parameters $p_{0}, q_{0}$ lie in the permitted range ] $1,+\infty\left[\right.$, whereas the first and the fourth inequality yield that $1-\frac{d}{2} \eta<0$, so that

$$
\sum_{l=1}^{+\infty}\left\|A_{m_{l}}\right\|_{L^{p}\left(\mathbb{R}^{d} ; \ell^{q}\right) \rightarrow L^{p}\left(\mathbb{R}^{d} ; \ell q\left(L^{\infty}(] 0,+\infty[)\right)\right)} \leqslant C(d, p, q) 2^{l\left(1-\frac{d}{2} \eta\right)}<+\infty,
$$

and consequently, appealing also to (1) and Proposition 11, we get

$$
\begin{aligned}
\left\|\left(\sum_{n=1}^{+\infty}\left|\mathcal{M}_{S} f_{n}(\cdot)\right|^{q}\right)^{\frac{1}{q}}\right\|_{L^{p}\left(\mathbb{R}^{d}\right)} & \leqslant\left(\left\|A_{m_{0}}\right\|_{L^{p}\left(\mathbb{R}^{d} ; \ell^{q}\right) \rightarrow L^{p}\left(\mathbb{R}^{d} ; \ell^{q}\left(L^{\infty}(] 0,+\infty[)\right)\right)}+\right. \\
& \left.\sum_{l=1}^{+\infty}\left\|A_{m_{l}}\right\|_{L^{p}\left(\mathbb{R}^{d} ; \ell^{q}\right) \rightarrow L^{p}\left(\mathbb{R}^{d} ; \ell^{q}\left(L^{\infty}(] 0,+\infty[)\right)\right)}\right)\left\|\left(\sum_{n=1}^{+\infty}\left|f_{n}(\cdot)\right|^{q}\right)^{\frac{1}{q}}\right\|_{L^{p}\left(\mathbb{R}^{d}\right)},
\end{aligned}
$$

and finally

$$
\left\|\left(\sum_{n=1}^{+\infty}\left|\mathcal{M}_{S} f_{n}(\cdot)\right|^{q}\right)^{\frac{1}{q}}\right\|_{L^{p}\left(\mathbb{R}^{d}\right)} \leqslant C(d, p, q)\left\|\left(\sum_{n=1}^{+\infty}\left|f_{n}(\cdot)\right|^{q}\right)^{\frac{1}{q}}\right\|_{L^{p}\left(\mathbb{R}^{d}\right)} .
$$




\section{Proof of Theorem 1}

This section is devoted to the proof of our first dimensionless result, that is Theorem 1. We shall use several auxiliary operators, and, even if we shall follow the same strategy as in the scalar case (see [9, 28,), we give complete detailed proofs for the reader's convenience.

Let us first introduce the following weighted maximal operator, depending on a parameter $k \in \mathbb{N}$,

$$
\mathcal{M}_{d, k} f(x)=\sup _{r>0} \frac{\int_{|y| \leqslant r}|f(x-y)||y|^{k} d y}{\int_{|y| \leqslant r}|y|^{k} d y}, \quad x \in \mathbb{R}^{d} .
$$

It is enough to take polar coordinates in the definition of $\mathcal{M}_{d, k}$ in order to obtain the following pointwise inequality

$$
\mathcal{M}_{d, k} f(x) \leqslant \mathcal{M}_{S}|f|(x), \quad x \in \mathbb{R}^{d} .
$$

Therefore, if we apply Theorem 2 we get that for $d \geqslant 3, d /(d-1)<p, q<d$ and every sequence $\left(f_{n}\right)_{n \geqslant 1}$ of measurable functions defined on $\mathbb{R}^{d}$ such that $\left(\sum_{n=1}^{+\infty}\left|f_{n}(\cdot)\right|^{q}\right)^{\frac{1}{q}} \in L^{p}\left(\mathbb{R}^{d}\right)$

$$
\left\|\left(\sum_{n=1}^{+\infty}\left|\mathcal{M}_{d, k} f_{n}(\cdot)\right|^{q}\right)^{\frac{1}{q}}\right\|_{L^{p}\left(\mathbb{R}^{d}\right)} \leqslant C(d, p, q)\left\|\left(\sum_{n=1}^{+\infty}\left|f_{n}(\cdot)\right|^{q}\right)^{\frac{1}{q}}\right\|_{L^{p}\left(\mathbb{R}^{d}\right)},
$$

where $C(d, p, q)$ is a constant independent of $k$ and $\left(f_{n}\right)_{n \geqslant 1}$. Now, we shall obtain Theorem 1 by lifting inequality (6) in lower dimension $d^{\prime}$ into $\mathbb{R}^{d}$ (with $d^{\prime} \leqslant d$ and $k=d-d^{\prime}$ ) by integrating over the Grassmannian of $d^{\prime}$-planes in $\mathbb{R}^{d}$. This method of descent is in the spirit of the Calderón-Zygmund method of rotations.

We therefore decompose $\mathbb{R}^{d}$ as follows $\mathbb{R}^{d}=\mathbb{R}^{d^{\prime}} \times \mathbb{R}^{d-d^{\prime}}$ and for $x \in \mathbb{R}^{d}$, we write $x=$ $\left(x_{d^{\prime}}, x_{d-d^{\prime}}\right)$ with $x_{d^{\prime}} \in \mathbb{R}^{d^{\prime}}$ and $x_{d-d^{\prime}} \in \mathbb{R}^{d-d^{\prime}}$. Besides, for all $\theta \in \mathcal{O}(d)=\left\{\theta^{\prime} \in \mathbb{R}^{d \times d}:\left|\theta^{\prime}(x)\right|=\right.$ $|x|$ for all $\left.x \in \mathbb{R}^{d}\right\}$ the orthogonal group, we introduce the following auxiliary operator

$$
\mathcal{M}_{d^{\prime}}^{\theta} f(x)=\sup _{r>0} \frac{\int_{\left|y_{d^{\prime}}\right| \leqslant r}\left|f\left(x-\theta\left(y_{d^{\prime}}, 0\right)\right)\right|\left|y_{d^{\prime}}\right|^{d-d^{\prime}} d y_{d^{\prime}}}{\int_{\left|y_{d^{\prime}}\right| \leqslant r}\left|y_{d^{\prime}}\right|^{d-d^{\prime}} d y_{d^{\prime}}}, \quad x \in \mathbb{R}^{d} .
$$

We shall need the following lemma, which provides us Fefferman-Stein inequalities for $\mathcal{M}_{d^{\prime}}^{\theta}$ with bound independent of $\theta$ and $d$.

Lemma 1. Let $d^{\prime} \geqslant 3$ and $d^{\prime} /\left(d^{\prime}-1\right)<p, q<d^{\prime}$. Let $\left(f_{n}\right)_{n \geqslant 1}$ be a sequence of measurable functions defined on $\mathbb{R}^{d}$. If $\left(\sum_{n=1}^{+\infty}\left|f_{n}(\cdot)\right|^{q}\right)^{\frac{1}{q}} \in L^{p}\left(\mathbb{R}^{d}\right)$, then we have

$$
\left\|\left(\sum_{n=1}^{+\infty}\left|\mathcal{M}_{d^{\prime}}^{\theta} f_{n}(\cdot)\right|^{q}\right)^{\frac{1}{q}}\right\|_{L^{p}\left(\mathbb{R}^{d}\right)} \leqslant C\left(d^{\prime}, p, q\right)\left\|\left(\sum_{n=1}^{+\infty}\left|f_{n}(\cdot)\right|^{q}\right)^{\frac{1}{q}}\right\|_{L^{p}\left(\mathbb{R}^{d}\right)},
$$

where $C\left(d^{\prime}, p, q\right)$ is a constant independent of $d, \theta$ and $\left(f_{n}\right)_{n \geqslant 1}$.

Proof. Since we have

$$
\left\|\left(\sum_{n=1}^{+\infty}\left|\mathcal{M}_{d^{\prime}}^{\theta} f_{n}(\cdot)\right|^{q}\right)^{\frac{1}{q}}\right\|_{L^{p}\left(\mathbb{R}^{d}\right)}^{p}=\left\|\left(\sum_{n=1}^{+\infty}\left|\mathcal{M}_{d^{\prime}}^{\mathrm{Id}}\left(f_{n} \circ \theta\right) \circ \theta^{-1}(\cdot)\right|^{q}\right)^{\frac{1}{q}}\right\|_{L^{p}\left(\mathbb{R}^{d}\right)}^{p},
$$

then we get by invariance

$$
\left\|\left(\sum_{n=1}^{+\infty}\left|\mathcal{M}_{d^{\prime}}^{\theta} f_{n}(\cdot)\right|^{q}\right)^{\frac{1}{q}}\right\|_{L^{p}\left(\mathbb{R}^{d}\right)}^{p}=\left\|\left(\sum_{n=1}^{+\infty}\left|\mathcal{M}_{d^{\prime}}^{\mathrm{Id}}\left(f_{n} \circ \theta\right)(\cdot)\right|^{q}\right)^{\frac{1}{q}}\right\|_{L^{p}\left(\mathbb{R}^{d}\right)}^{p}
$$


which is equivalent to

$$
\begin{aligned}
& \left\|\left(\sum_{n=1}^{+\infty}\left|\mathcal{M}_{d^{\prime}}^{\theta} f_{n}(\cdot)\right|^{q}\right)^{\frac{1}{q}}\right\|_{L^{p}\left(\mathbb{R}^{d}\right)}^{p} \\
& \quad=\int_{\mathbb{R}^{d-d^{\prime}}}\left[\int_{\mathbb{R}^{d^{\prime}}}\left(\sum_{n=1}^{+\infty}\left|\mathcal{M}_{d^{\prime}, d-d^{\prime}}\left(f_{n} \circ \theta \circ g_{x_{d-d^{\prime}}}\right)\left(x_{d}^{\prime}\right)\right|^{q}\right)^{\frac{p}{q}} d x_{d^{\prime}}\right] d x_{d-d^{\prime}}
\end{aligned}
$$

where we have set

$$
g_{x_{d-d^{\prime}}}: \mathbb{R}^{d^{\prime}} \rightarrow \mathbb{R}^{d}, \quad x_{d^{\prime}} \mapsto\left(x_{d^{\prime}}, x_{d-d^{\prime}}\right) .
$$

If we now apply inequality (6), we are led to

$$
\begin{aligned}
& \left\|\left(\sum_{n=1}^{+\infty}\left|\mathcal{M}_{d^{\prime}}^{\theta} f_{n}(\cdot)\right|^{q}\right)^{\frac{1}{q}}\right\|_{L^{p}\left(\mathbb{R}^{d}\right)}^{p} \leqslant \\
& \qquad\left(d^{\prime}, p, q\right) \int_{\mathbb{R}^{d-d^{\prime}}}\left[\int_{\mathbb{R}^{d^{\prime}}}\left(\sum_{n=1}^{+\infty}\left|\left(f_{n} \circ \theta \circ g_{x_{d-d^{\prime}}}\right)\left(x_{d}^{\prime}\right)\right|^{q}\right)^{\frac{p}{q}} d x_{d^{\prime}}\right] d x_{d-d^{\prime}}
\end{aligned}
$$

and the expected result easily follows.

We shall also need the following lemma, which relates the Hardy-Littlewood maximal operator to the operator $\mathcal{M}_{d^{\prime}}^{\theta}$.

Lemma 2. If we denote by $d \mu$ the normalized Haar measure on $\mathcal{O}(d)$, then we have the following pointwise inequality

$$
\mathcal{M} f(x) \leqslant \int_{\mathcal{O}(d)} \mathcal{M}_{d^{\prime}}^{\theta} f(x) d \mu(\theta), \quad x \in \mathbb{R}^{d} .
$$

Proof. If we prove the following equality

$$
\frac{\int_{|y| \leqslant r}|f(y)| d y}{\int_{|y| \leqslant r} d y}=\frac{\int_{\mathcal{O}(d)} \int_{\left|y_{d^{\prime}}\right| \leqslant r}\left|f\left(\theta\left(y_{d^{\prime}}, 0\right)\right)\right|\left|y_{d^{\prime}}\right|^{d-d^{\prime}} d y_{d^{\prime}} d \mu(\theta)}{\int_{\left|y_{d^{\prime}}\right| \leqslant r}\left|y_{d^{\prime}}\right|^{d-d^{\prime}} d y_{d^{\prime}}},
$$

then the expected inequality follows easily. Indeed, the previous equality allows us to write

$$
\begin{aligned}
\frac{1}{|B(0, r)|} \int_{B(0, r)}|f(x-y)| d y & =\frac{\int_{\mathcal{O}(d)} \int_{\left|y_{d^{\prime}}\right| \leqslant r} \mid f\left(\left.\left(x-\theta\left(y_{d^{\prime}}, 0\right)\right)|| y_{d^{\prime}}\right|^{d-d^{\prime}} d y_{d^{\prime}} d \mu(\theta)\right.}{\int_{\left|y_{d^{\prime}}\right| \leqslant r}\left|y_{d^{\prime}}\right|^{d-d^{\prime}} d y_{d^{\prime}}} \\
& \leqslant \int_{\mathcal{O}(d)} \mathcal{M}_{d^{\prime}}^{\theta} f(x) d \mu(\theta)
\end{aligned}
$$

and it is then enough to take the supremum over all $r>0$. Therefore, we are left with the task of establishing (7). Of course, by density arguments, we can restrict ourselves to finite linear combinations of functions whose expression has the following form $f(x)=f_{0}(|x|) f_{1}\left(x^{\prime}\right)$, with $x=|x| x^{\prime}$ and $x^{\prime} \in S^{d-1}$. If we take polar coordinates in the left-side of (7) we get

$$
\frac{d}{r^{d}} \int_{0}^{r} f_{0}(t) t^{d-1} d t \int_{S^{d-1}} f_{1}\left(y^{\prime}\right) d \sigma\left(y^{\prime}\right)
$$

and if we take polar coordinates in the right-side of (7) we get

$$
\frac{d}{r^{d}} \int_{0}^{r} f_{0}(t) t^{d-1} d t \int_{\mathcal{O}(d)} \int_{S^{d^{\prime}-1}} f_{1}\left(\theta\left(y_{d^{\prime}}^{\prime}, 0\right)\right) d \sigma\left(y_{d^{\prime}}^{\prime}\right) d \mu(\theta) .
$$

Thus, we only have to prove the following equality

$$
\int_{S^{d-1}} f_{1}\left(y^{\prime}\right) d \sigma\left(y^{\prime}\right)=\int_{\mathcal{O}(d)} \int_{S^{d^{\prime}-1}} f_{1}\left(\theta\left(y_{d^{\prime}}^{\prime}, 0\right)\right) d \sigma\left(y_{d^{\prime}}^{\prime}\right) d \mu(\theta) .
$$


Let us note that we can write

$$
\int_{\mathcal{O}(d)} \int_{S^{d^{\prime}-1}} f_{1}\left(\theta\left(y_{d^{\prime}}^{\prime}, 0\right)\right) d \sigma\left(y_{d^{\prime}}^{\prime}\right) d \mu(\theta)=\int_{S^{d-1}} f_{1}(\eta) d \nu(\eta),
$$

where we have used the notation $d \nu$ to denote the pushforward of $d \sigma \otimes d \mu$ under the map $\left(y_{d^{\prime}}^{\prime}, \tau\right) \mapsto \theta\left(y_{d^{\prime}}^{\prime}, 0\right)$. Since $d \nu$ is invariant under the left-action of $\mathcal{O}(d)$, we claim that $d \nu=d \sigma$, and the proof is finished.

With the two previous lemmas in mind, we are now in a position to prove Theorem 1 .

Proof of Theorem 1, Let $1<p, q<+\infty$.

If $d \leqslant 3$ or $d \leqslant \max \left(\frac{p}{p-1}, \frac{q}{q-1}\right)$ or $d \leqslant \max (p, q)$, there is nothing to do, that is, we invoke the standard methods to get Fefferman-Stein inequalities.

We can therefore assume that $d \geqslant 3$ and that $\frac{d}{d-1}<p, q<d$. Thus, we write $d=d^{\prime}+\left(d-d^{\prime}\right)$ with

$$
d^{\prime}=\lfloor\max (2, p, q, p /(p-1), q /(q-1))\rfloor+1 .
$$

The expected result then follows easily by using both Lemma 1 and Lemma 2.

\section{Proof of Theorem 3}

This section is devoted to the proof of the dimension free estimate of the UMD lattice valued Hardy-Littlewood maximal operator. In order to make the meaning of the latter precise, we recall the necessary background on UMD lattices, i.e. Banach lattices which are UMD spaces. First, for a general treatment of Banach lattices and their geometric properties, we refer the reader to Chapter 1 in [19]. Second, a Banach space $Y$ is called UMD space if the Hilbert transform

$$
H: L^{p}(\mathbb{R}) \rightarrow L^{p}(\mathbb{R}), H f(x)=P V-\int_{\mathbb{R}} \frac{1}{x-y} f(y) d y,
$$

extends to a bounded operator on $L^{p}(\mathbb{R} ; Y)$, for some (equivalently for all) $1<p<+\infty$, see Theorem 5.1 in [16]. A UMD space is super-reflexive [1], and hence (almost by definition) Bconvex. Let in the following $Y$ be a UMD space which is also a Banach lattice. By $B$-convexity, $Y$ is order continuous and therefore it can be represented as a lattice consisting of (equivalence classes of) measurable functions on some measure space $(\Omega, \mu)$, see 1.a, 1.b in [19]. If $Y$ is a UMD lattice and $1<p<+\infty$, then $L^{p}\left(\mathbb{R}^{d} ; Y\right)$ is again a UMD lattice.

Remark. In the proof below, we will use frequently and tacitly the following almost trivial observation: if $0 \leqslant M(f) \leqslant N(f)$ for some element $f \in L^{p}\left(\mathbb{R}^{d} ; Y\right)$ and images $M(f), N(f)$ of (typically non-linear) mappings $M, N$, belonging to $L^{p}\left(\mathbb{R}^{d} ; Y\right)$, then $\|M(f)\|_{L^{p}\left(\mathbb{R}^{d} ; Y\right)} \leqslant\|N(f)\|_{L^{p}\left(\mathbb{R}^{d} ; Y\right)}$. This follows promptly from lattice axioms.

Since the UMD lattice $Y$ is order continuous, by Proposition 1.a.8 in [19, it is also order complete. Now define the UMD lattice valued Hardy-Littlewood maximal operator and spherical maximal operator by

$$
\mathcal{M} f(x, \cdot)=\sup _{r>0} \frac{1}{|B(x, r)|} \int_{B(x, r)}|f(y, \cdot)| d y, \quad f \in L^{p}\left(\mathbb{R}^{d}\right) \otimes Y \subseteq L^{p}\left(\mathbb{R}^{d} ; Y\right)
$$

and

$$
\mathcal{M}_{S} f(x, \cdot)=\sup _{r>0}\left|\int_{S^{d-1}} f(x-r y, \cdot) d \sigma(y)\right|, \quad f \in \mathcal{S}\left(\mathbb{R}^{d}\right) \otimes Y \subseteq L^{p}\left(\mathbb{R}^{d} ; Y\right) .
$$

Using the order completeness of $L^{p}\left(\mathbb{R}^{d} ; Y\right)$ and the scalar valued boundedness of $\mathcal{M}$ and $\mathcal{M}_{S}$, it is not difficult to show that the above suprema exist in $L^{p}\left(\mathbb{R}^{d} ; Y\right)$ in the lattice sense. Thus, $\mathcal{M} f\left(\right.$ resp. $\left.\mathcal{M}_{S} f\right)$ are well-defined elements of $L^{p}\left(\mathbb{R}^{d} ; Y\right)$ for the above $f$ and $1<p<+\infty$ 
(resp. $\frac{d}{d-1}<p<+\infty$ ). In the sequel, we can restrict in statements of boundedness of maximal operators first to $f$ belonging to $L^{p}\left(\mathbb{R}^{d}\right) \otimes Y$ or to $\mathcal{S}\left(\mathbb{R}^{d}\right) \otimes Y$ to have a priori maximal functions belonging to $L^{p}\left(\mathbb{R}^{d} ; Y\right)$. Then use the remark above in this section and get an inequality for such $f$

$$
\|\mathcal{M} f\|_{L^{p}\left(\mathbb{R}^{d} ; Y\right)} \leqslant C\|f\|_{L^{p}\left(\mathbb{R}^{d} ; Y\right)}
$$

resp. with $\mathcal{M}$ on the l.h.s. replaced by $\mathcal{M}_{S}$, or the several other maximal operators we use in the proof. Finally for general $f \in L^{p}\left(\mathbb{R}^{d} ; Y\right)$ and an approximating sequence of $f$, say $\left(f_{n}\right)_{n \geqslant 1}$ belonging to $\mathcal{S}\left(\mathbb{R}^{d}\right) \otimes Y, \mathcal{M} f$ can be well defined by $\mathcal{M} f=\lim _{n} \mathcal{M} f_{n}$, due to

$$
\left\|\mathcal{M} f_{n}-\mathcal{M} f_{m}\right\| \leqslant\left\|\mathcal{M}\left(f_{n}-f_{m}\right)\right\| \leqslant C\left\|f_{n}-f_{m}\right\| .
$$

We thus end up with the desired estimate $\|\mathcal{M} f\| \leqslant C\|f\|$ for all $f \in L^{p}\left(\mathbb{R}^{d} ; Y\right)$, with the same constant $C$ as before.

Proof of Theorem [3. Let $1<p<+\infty$. Since $Y$ is a UMD Banach lattice, say over the measure space $(\Omega, \mu)$, we claim, invoking Corollary page 216 in [24, that there exists another UMD Banach lattice $Z$ defined on $(\Omega, \mu)$, such that

$$
Y=[Z, H]_{\eta}, \quad \text { with } H=L^{2}(\Omega, \mu) \text { a Hilbert space, }
$$

where $[\cdot, \cdot]_{\eta}$ is the complex interpolation method, with $\left.\eta \in\right] 0,1[$. Having a closer look at the proof of Corollary page 216 in [24] (alternatively, by replacing $Z$ with $[Z, H]_{\eta_{0}}$ and using reiteration of complex interpolation), we can assume $\eta$ sufficiently close to 0 such that $\frac{\eta}{2}<\frac{1}{p}<1-\frac{\eta}{2}$.

Let $\mathcal{M}_{S}$ be the spherical maximal operator. Our first aim is to show its boundedness on $L^{p}\left(\mathbb{R}^{d} ; Y\right)$ for $d$ sufficiently large. Write

$$
\mathcal{M}_{S} f(x, y) \leqslant \sum_{l=0}^{+\infty} M_{m_{l}} f(x, y)
$$

as in (11), as soon as $d \geq 3$. Here and in the sequel, $x \in \mathbb{R}^{d}$ and $y \in \Omega$. For $X \in\{Z, H, Y\}$, we denote the linear operator

$$
A_{m_{l}}:\left\{\begin{array}{l}
L^{p}\left(\mathbb{R}^{d} ; X\right) \rightarrow L^{p}\left(\mathbb{R}^{d} ; X\left(L^{\infty}(] 0,+\infty[)\right)\right) \\
f \mapsto f *\left(m_{l}^{\vee}\right)_{r}(x, y) .
\end{array}\right.
$$

We clearly have the following equalities

$$
\begin{aligned}
M_{m_{l}} f(x, y) & =\left\|A_{m_{l}} f(x, y, \cdot)\right\|_{L^{\infty}(] 0,+\infty[)} \\
\left\|M_{m_{l}} f\right\|_{L^{p}\left(\mathbb{R}^{d} ; X\right)} & =\left\|A_{m_{l}} f\right\|_{L^{p}\left(\mathbb{R}^{d} ; X\left(L^{\infty}(] 0,+\infty[)\right)\right)} .
\end{aligned}
$$

Moreover, we have

$$
M_{m_{0}} f(x, y) \leqslant C(d) \mathcal{M} f(x, y),
$$

and it is a well-known fact that the centered Hardy-Littlewood maximal operator satisfies

$$
\mathcal{M} f(x) \leqslant C(d) \mathcal{M}_{\Delta}|f|(x),
$$

where we have set

$$
\mathcal{M}_{\Delta} f(x)=\sup _{t>0}\left|e^{t \Delta} f(x)\right|
$$

for the maximal operator associated with the heat (diffusion) semigroup on $\mathbb{R}^{d}$. Then according to Theorem 2 in [30], $\mathcal{M}$ is bounded on $L^{p}\left(\mathbb{R}^{d} ; X\right)$ for any UMD Banach lattice $X$. We shall apply this fact with $X=Y$ and $X=Z$.

Namely, first, we have by the above

$$
\left\|M_{m_{0}} f\right\|_{L^{p}\left(\mathbb{R}^{d} ; Y\right)} \leqslant C(d, p, Y)\|f\|_{L^{p}\left(\mathbb{R}^{d} ; Y\right)} .
$$


Since $H$ is a Hilbert space, we have by Proposition 3 the inequality

$$
\left\|A_{m_{l}} f\right\|_{L^{2}\left(\mathbb{R}^{d} ; H\left(L^{\infty}(] 0,+\infty[)\right)\right)} \leqslant \frac{C(d)}{2^{\frac{l(d-2)}{2}}}\|f\|_{L^{2}\left(\mathbb{R}^{d} ; H\right)} .
$$

Furthermore, according to (3), we have

$$
\sup _{r>0}\left|A_{m_{l}} f(x, y, r)\right| \leqslant C(d) 2^{l} \mathcal{M} f(x, y),
$$

and thus,

$$
\left\|A_{m_{l}} f\right\|_{L^{p_{0}\left(\mathbb{R}^{d} ; Z\left(L^{\infty}(] 0,+\infty[)\right)\right)}} \leqslant C\left(d, p_{0}, Z\right) 2^{l}\|f\|_{L^{p_{0}\left(\mathbb{R}^{d} ; Z\right)},},
$$

for $\left.p_{0} \in\right] 1,+\infty[$ to be chosen later. We next want to apply complex interpolation of (9) and (10). To this end, note that

$$
\left[Z\left(\mathrm{~L}^{\infty}\right), H\left(L^{\infty}\right)\right]_{\eta} \hookrightarrow[Z, H]_{\eta}\left(L^{\infty}\right)=Y\left(L^{\infty}\right)
$$

contractively, according for instance to (1.1) in [11] and Calderón's interpolation identification between the Calderón-Lozanovskii space $Z^{1-\eta} H^{\eta}$ and $[Z, H]_{\eta}=Y$, see for instance page 215 in 24]. Then we get

$$
\left\|A_{m_{l}} f\right\|_{L^{p}\left(\mathbb{R}^{d} ; Y\left(L^{\infty}(] 0,+\infty[)\right)\right)} \leqslant C(d, p, \eta, Z) 2^{l k(\eta)}\|f\|_{L^{p}\left(\mathbb{R}^{d} ; Y\right)},
$$

with

$$
\frac{1}{p}=\frac{\eta}{2}+\frac{1-\eta}{p_{0}}=\frac{1}{p_{0}}+\eta\left(\frac{1}{2}-\frac{1}{p_{0}}\right)
$$

and

$$
k(\eta)=-\eta \frac{d-2}{2}+(1-\eta)=1-\eta \frac{d}{2} .
$$

Here, since $\eta$ was sufficiently close to 0 , there exists an appropriate choice of $\left.p_{0} \in\right] 1,+\infty[$. If now $d>d_{0}:=\max (3,\lfloor 2 / \eta\rfloor+1)$, then $k(\eta)<0$, so that then

$$
\left\|\mathcal{M}_{S} f\right\|_{L^{p}\left(\mathbb{R}^{d} ; Y\right)} \leqslant
$$

$$
\left\|A_{m_{0}} f\right\|_{L^{p}\left(\mathbb{R}^{d} ; Y\left(L^{\infty}(] 0,+\infty[)\right)\right)}+\sum_{l=1}^{+\infty} C(d, p, \eta, Z) 2^{l k(\eta)}\|f\|_{L^{p}\left(\mathbb{R}^{d} ; Y\right)},
$$

which yields to

$$
\left\|\mathcal{M}_{S} f\right\|_{L^{p}\left(\mathbb{R}^{d} ; Y\right)} \leqslant C^{\prime}(d, p, Y)\|f\|_{L^{p}\left(\mathbb{R}^{d} ; Y\right)} .
$$

We deduce that for $d^{\prime} \geqslant d_{0}$

$$
\left\|\mathcal{M}_{d^{\prime}}^{\theta}\right\|_{L^{p}\left(\mathbb{R}^{d} ; Y\right)} \leqslant C\left(d^{\prime}, p, Y\right)\|f\|_{L^{p}\left(\mathbb{R}^{d} ; Y\right)},
$$

which can be proved as Lemma 1 using (5), and the above vector-valued boundedness of the spherical maximal operator, i.e. on $L^{p}\left(\mathbb{R}^{d^{\prime}} ; Y\right)$. Then by Lemma 2, as in the case $Y=\ell^{q}$, we deduce with $d^{\prime}=d_{0}$ and $d \geqslant d^{\prime}$ that the centered Hardy-Littlewood maximal operator is bounded on $L^{p}\left(\mathbb{R}^{d} ; Y\right)$, with bound $C\left(d^{\prime}, p, Y\right)$ independent of $d \geqslant d^{\prime}$.

For dimensions $d<d^{\prime}$, we invoke the above explained estimate of $\mathcal{M} f(x) \leqslant C(d) \mathcal{M}_{\Delta}|f|(x)$. 


\section{Applichtion to the Grushin maximal operator}

In this section, we show that Theorems 1 and 3 can be transferred to the context of Grushin operators. Initially studied by Grushin (see for example [15]), these operators have received considerable attention in recent times, especially with some results on their harmonic analysis, see for instance [10, 18, 21]. Moreover, dimensionless type results have been in particular investigated, mainly for Riesz transforms associated with them, see [25].

Let us recall the setting. The Grushin operator is given by

$$
\Delta_{G}=\sum_{i=1}^{d} \frac{\partial^{2}}{\partial x_{i}^{2}}+|x|^{2} \frac{\partial^{2}}{\partial u^{2}}=\sum_{i=1}^{d}\left(X_{i}^{2}+U_{i}^{2}\right)
$$

on the space $\mathbb{R}^{d+1}=\mathbb{R}_{x}^{d} \times \mathbb{R}_{u}$, with

$$
|x|^{2}=\sum_{i=1}^{d} x_{i}^{2}, \quad X_{i}=\frac{\partial}{\partial x_{i}}, \quad U_{i}=x_{i} \frac{\partial}{\partial u},
$$

where the smooth vector fields $\left\{X_{i}, U_{i}\right\}_{1 \leq i \leq d}$ satisfy the Hörmander condition. We point out that the operator $\Delta_{G}$ is related to the Heisenberg group $\mathbb{H}_{d}$, since it is actually the image of a sub-Laplacian associated with $\mathbb{H}_{d}$ under a representation acting on functions on $\mathbb{R}^{d+1}$. Let $d_{C C}$ denote the Carnot-Carathéodory distance associated with $\left\{X_{1}, \ldots, X_{d}, U_{1}, \ldots, U_{d}\right\}$ (see for example [29]). Then $\left(\mathbb{R}^{d+1}, d_{C C}, d m\right)$ is a space of homogeneous type, where $d m$ stands for the Lebesgue measure, which is not, however, translation invariant. We define a further pseudometric on $\mathbb{R}^{d+1}$. Namely, for $g=(x, u)$ and $g^{\prime}=\left(x^{\prime}, u^{\prime}\right)$ belonging to $\mathbb{R}_{x}^{d} \times \mathbb{R}_{u}$, we let

$$
d_{K}\left(g, g^{\prime}\right)=\sqrt{\sqrt{\left(|x|^{2}+\left|x^{\prime}\right|^{2}\right)^{2}+\left(2\left|u-u^{\prime}\right|\right)^{2}}-2\left\langle x, x^{\prime}\right\rangle},
$$

where $\langle\cdot, \cdot\rangle$ denotes the standard Euclidean scalar product. Then $d_{K}$ is a pseudo-distance on $\mathbb{R}^{d+1}$ (which is, in fact, equivalent to $d_{C C}$ [18]) related to the fundamental solution of $\Delta_{G}$ (that is to say Green's function). We denote balls with respect to these two (pseudo)-distances by

$$
B_{C C}(g, r)=\left\{g^{\prime} \in \mathbb{R}^{d+1}: d_{C C}\left(g, g^{\prime}\right) \leqslant r\right\}
$$

and

$$
B_{K}(g, r)=\left\{g^{\prime} \in \mathbb{R}^{d+1}: d_{K}\left(g, g^{\prime}\right) \leqslant r\right\} .
$$

This gives rise to the following Hardy-Littlewood maximal operators $\mathcal{M}_{C C}$ and $\mathcal{M}_{K}$, respectively and naturally given for $f \in L_{\text {loc }}^{1}\left(\mathbb{R}^{d+1}\right)$ by

$$
\begin{array}{ll}
\mathcal{M}_{C C} f(g)=\sup _{r>0} \frac{1}{\left|B_{C C}(g, r)\right|} \int_{B_{C C}(g, r)}\left|f\left(g^{\prime}\right)\right| d g^{\prime}, & g \in \mathbb{R}^{d+1}, \\
\mathcal{M}_{K} f(g)=\sup _{r>0} \frac{1}{\left|B_{K}(g, r)\right|} \int_{B_{K}(g, r)}\left|f\left(g^{\prime}\right)\right| d g^{\prime}, & g \in \mathbb{R}^{d+1} .
\end{array}
$$

If $Y=Y(\Omega)$ is a UMD Banach lattice, these operators extend, for $g \in \mathbb{R}^{d+1}, \omega \in \Omega, f \in$ $L^{p}\left(\mathbb{R}^{d+1}\right) \otimes Y$, by the formula

$$
\left(\mathcal{M}_{C C} f\right)(g, \omega)=\mathcal{M}_{C C}(f(\cdot, \omega))(g),
$$

and similarly for $\mathcal{M}_{K}$. This a priori definition of $\mathcal{M}_{C C} f$ and $\mathcal{M}_{K} f$ yields a well-defined element in $L^{p}\left(\mathbb{R}^{d+1} ; Y\right)$, similarly to the remarks at the beginning of Section 5 concerning the HardyLittlewood maximal operator. We can also restrict ourselves to $f \in L^{p}\left(\mathbb{R}^{d}\right) \otimes Y$ in the proof of Theorem 4, to which we proceed now. 
Proof of Theorem 4. For $\mathcal{M}_{K}$, all we have to know is that (see (7.2) in [18])

$$
\mathcal{M}_{K} f(x, u) \leqslant C \mathcal{M}_{\mathbb{R}^{d}}\left(\mathcal{M}_{\mathbb{R}} f(\cdot, u)\right)(x),
$$

where $\mathcal{M}_{\mathbb{R}^{d}}$ and $\mathcal{M}_{\mathbb{R}}$ stand respectively for the standard Hardy-Littlewood maximal operator on $\mathbb{R}^{d}$ and $\mathbb{R}$. We can therefore apply the dimension free Theorems 1 and 3

Then for $\mathcal{M}_{C C}$, it suffices to have in mind that for all $g, g^{\prime} \in \mathbb{R}^{d+1}$ (see Propositions 5.1 and 5.2 in [18])

$$
d_{K}\left(g, g^{\prime}\right) \leqslant d_{C C}\left(g, g^{\prime}\right), \quad\left|B_{C C}(g, 1)\right| \geqslant C\left|B_{K}(g, 1)\right|
$$

with a constant $C>0$ independent of the dimension $d \in \mathbb{N}$. Indeed, using for both $B=B_{C C}$ and $B=B_{K}$

$$
|B((x, u), r)|=r^{d+2}\left|B\left(\delta_{r^{-1}}(x, u), 1\right)\right|
$$

where $\delta_{r^{-1}}(x, u)=\left(r^{-1} x, r^{-2} u\right)$ [18], we deduce

$$
\begin{aligned}
\mathcal{M}_{C C} f(g) & =\sup _{r>0} \frac{1}{\left|B_{C C}(g, r)\right|} \int_{B_{C C}(g, r)}\left|f\left(g^{\prime}\right)\right| d g^{\prime} \\
& =\sup _{r>0} \frac{1}{r^{d+2}\left|B_{C C}\left(\delta_{r^{-1}} g, 1\right)\right|} \int_{B_{C C}(g, r)}\left|f\left(g^{\prime}\right)\right| d g^{\prime} \\
& \leqslant \sup _{r>0} \frac{1}{C r^{d+2}\left|B_{K}\left(\delta_{r^{-1}} g, 1\right)\right|} \int_{B_{K}(g, r)}\left|f\left(g^{\prime}\right)\right| d g^{\prime},
\end{aligned}
$$

that is to say,

$$
\mathcal{M}_{C C} f(g) \leqslant \frac{1}{C} \mathcal{M}_{K} f(g) .
$$

Therefore, the statement for $\mathcal{M}_{C C}$ follows from that for $\mathcal{M}_{K}$.

Another interesting case would be the centered Hardy-Littlewood maximal operator on the Heisenberg group, as studied e.g. in [17, 31. After personal communication with Hong-Quan Li, we do not know whether this maximal operator admits dimension free $\ell^{q}$ or UMD lattice valued estimates.

Acknowledgments. The authors wish to thank the referee for her/his careful reading of the manuscript and helpful comments and remarks which improved the quality of the paper.

\section{REFERENCES}

[1] Aldous, D. Unconditional bases and martingales in $L_{p}(F)$ Math. Proc. Cambridge Philos. Soc. 85 (1979), no. $1,117-123$

[2] Andrews, G.E., Askey, R. and Roy, R. Special functions Encyclopedia of Mathematics and its Applications, 71. Cambridge University Press, Cambridge, 1999.

[3] Bourgain, J. On high dimensional maximal functions associated to convex bodies Amer. J. Math. 108 (1986), no. $6,1467-1476$.

[4] Bourgain, J. Averages in the plane over convex curves and maximal operators J. Analyse Math. 47 (1986), 69-85.

[5] Bourgain, J. On the $L^{p}$-bounds for maximal functions associated to convex bodies in $\mathbb{R}^{n}$ Israel J. Math. 54 (1986), no. 3, 257-265.

[6] Bourgain, J. On dimension free maximal inequalities for convex symmetric bodies in $\mathbb{R}^{n}$ Geometrical aspects of functional analysis (1985/86), 168-176, Lecture Notes in Math. 1267, Springer, Berlin, 1987.

[7] Bourgain, J. On the Hardy-Littlewood maximal function for the cube Israel J. Math. 203 (2014), no. 1, 275-293.

[8] Carbery, A. An almost-orthogonality principle with applications to maximal functions associated to convex bodies Bull. Amer. Math. Soc. (N.S.) 14 (1986), no. 2, 269-273.

[9] Deleaval, L., Guédon, O. and Maurey, B. Dimension free bounds for the Hardy-Littlewood maximal operator associated to convex sets Ann. Fac. Sci. Toulouse Math., in press. 
[10] Dziubański, J. and Jotsaroop, K. On Hardy and BMO spaces for Grushin operator J. Fourier Anal. Appl. 22 (2016), 954-995.

[11] Fan, M. On interpolation of vector-valued Banach lattices and Calderón-Lozanovskii construction Math. Nachr. 227 (2001), 63-80.

[12] Fefferman, C. and Stein, E.M. Some maximal inequalities Amer. J. Math. 93 (1971), 107-115.

[13] Garciá-Cuerva, J. and Rubio de Francia, J.L. Weighted Norm Inequalities and Related Topics North-Holland Mathematics Studies, 116. North-Holland Publishing Co., Amsterdam, 1985.

[14] Grafakos, L. Classical Fourier analysis Second Edition, Graduate Texts in Mathematics, 249. Springer, New York, 2008.

[15] Grušin, V.V. A certain class of elliptic pseudodifferential operators that are degenerate on a submanifold Mat. Sb. (N.S.) 84 (1971), no. 126, 163-195.

[16] Hytönen, T., van Neerven, J., Veraar, M. and Weis, L. Analysis in Banach spaces I, Martingales and Littlewood-Paley theory Preprint available on http://fa.its.tudelft.nl/ neerven/

[17] Li, H.-Q. Fonctions maximales centrées de Hardy-Littlewood sur les groupes de Heisenberg Studia Math. 191 (2009), no. 1, 89-100.

[18] Li, H.-Q. Fonctions maximales centrées de Hardy-Littlewood pour les opérateurs de Grushin Preprint on arXiv:1207.3128.

[19] Lindenstrauss, J. and Tzafriri, L. Classical Banach spaces. II Function spaces. Results in Mathematics and Related Areas, 97. Springer-Verlag, Berlin-New York, 1979.

[20] Manna, R. Weighted inequalities for spherical maximal operator Proc. Japan Acad. Ser. A Math. Sci. 91 (2015), no. 9, 135-140.

[21] Martini, A. and Müller, D. A sharp multiplier theorem for Grushin operators in arbitrary dimensions Rev. Math. Iberoam. 30 (2014), 1265-1280.

[22] Müller, D. A geometric bound for maximal functions associated to convex bodies Pacific J. Math. 142 (1990), no. 2, 297-312.

[23] Rubio de Francia, J.L. Maximal functions and Fourier transforms Duke Math. J. 53 (1986), no. 2, 395-404.

[24] Rubio de Francia, J.L. Martingale and integral transforms of Banach space valued functions Lecture Notes in Math. 1221, Springer, Berlin, 1986.

[25] Sanjay, P.K. and Thangavelu, S. Dimension free boundedness of Riesz transforms for the Grushin operators Proc. Amer. Math. Soc. 142 (2014), no. 11, 3839-3851.

[26] Stein, E.M. Maximal functions. I. Spherical means Proc. Nat. Acad. Sci. U.S.A. 73 (1976), no. 7, $2174-2175$.

[27] Stein, E.M. The development of square functions in the work of A. Zygmund Bull. Amer. Math. Soc. (N.S.) 7 (1982), no. 2, 359-376.

[28] Stein, E.M. and Strömberg, J.-O. Behavior of maximal functions in $\mathbf{R}^{n}$ for large $n$ Ark. Mat. 21 (1983), no. 2, 259-269.

[29] Varopoulos, N.Th., Saloff-Coste, L. and Coulhon, T. Analysis and geometry on groups Cambridge Tracts in Mathematics, 100. Cambridge University Press, Cambridge, 1992.

[30] Xu, Q. $H^{\infty}$ functional calculus and maximal inequalities for semigroups of contractions on vector-valued $L_{p}$-spaces Int. Math. Res. Not. IMRN 14 (2015), 5715-5732.

[31] Zienkiewicz, J. Estimates for the Hardy-Littlewood maximal function on the Heisenberg group Colloq. Math. 103 (2005), 199-205.

Luc Deleaval

Laboratoire d'Analyse et de Mathématiques Appliquées (UMR 8050)

Université Paris-Est Marne la Vallée

5, Boulevard Descartes

Champs sur Marne

77454 Marne la Vallée Cedex 2

luc.deleaval@u-pem.fr

Christoph Kriegler

Laboratoire de Mathématiques (UMR 6620)

Université Blaise-Pascal (Clermont-Ferrand 2)

Campus Universitaire des Cézeaux

3 , place Vasarely

TSA 60026

CS 60026 
63178 Aubière Cedex, France

christoph.kriegler@math.univ-bpclermont.fr 